INTERNATIONAL DESIGN CONFERENCE - DESIGN 2018

https://doi.org/10.21278/idc.2018.0271

\title{
A NEW MODEL FOR CAPTURING DESIGN INFORMATION WITH AN AIM TO AID CHANGE PROPAGATION ASSESSMENT AND SUBSEQUENT REDESIGN
}

\author{
A. Brahma and D. C. Wynn
}

\begin{abstract}
A new model is developed to investigate the causes of change propagation and guide the redesign process. The model offers a micro-level perspective on the mechanisms through which changes propagate or are absorbed. Extending the Information Structure Framework (ISF) reported by Ahmad et al. (2013), the model uses a multi-domain approach combined with a reformulated detail design process layer. It is illustrated through a desk-based case study of routine mechanical design. Advantages and limitations are discussed along with possibilities for further work.
\end{abstract}

Keywords: engineering change, change management, detail design, information structure framework, design history

\section{Introduction}

The feasibility of a proposed design change is often evaluated by comparing the estimated cost with the benefits of the proposed changes. When a change is proposed, the potential for knock-on effect on other parts needs to be checked to avoid unforeseen issues. Although the difficulty of estimating change impact is a recognised problem, a solution is elusive (Huang and Mak, 1999). One reason is that in collaborative design none of the engineers are capable of seeing the product in its entirety in depth (Clarkson et al., 2004). The effect of engineering changes has been quoted as high as $30-50 \%$ of engineering capacity, cost and schedule (Terwiesch and Loch, 1999; Fricke et al., 2000). There is therefore a need to develop our understanding of how and why change propagates during redesign, and use that understanding to develop improved support tools.

This paper contributes towards a new model to assist in capturing design information relevant to change propagation, in order to investigate the causes of change propagation in detail and ultimately inform the development of pragmatic support. It complements the Information Structure Framework (ISF) (Ahmad et al., 2013), which is a multi-domain approach developed to help designers to assess change propagation and that may also assist the subsequent redesign process. The new model aims to address limitations of the ISF that were highlighted by Ahmad et al. (2013), in particular reporting on further research to develop its detail design layer. In this paper, the detail design layer of the ISF is reformulated and the new model is applied to a test case. Future research opportunities are discussed.

\section{Background}

Numerous methods have been proposed to predict change propagation. Some models are predictive and take a probabilistic approach, while others are deterministic and aim to provide more detailed 
design guidance. Some models focus on a single information domain, while others use multiple domains:

\subsection{Probabilistic predictive models vs. deterministic guidance models}

Predictive models tend to be mathematical in nature and are generally associated with the idea of quantification of cause and impact of change propagation in terms of probability or risk. For example, the Change Prediction Method (CPM) (Clarkson et al., 2004) uses a Design Structure Matrix (DSM) to represent linkages between different components/subsystems of a product. The CPM model is based on estimating the likelihood of change propagating from any component/subsystem to any other. Assigning these values relies on the expertise of engineers and hence the method involves unavoidable subjectivity. Predictive models have been used to aid decision-making not only in change management but also in risk assessment, and also to support project/product viability studies. Deterministic models give more specific results in terms of which parameters are affected and which components are subject to change. Examples include DCAM, an ontology-based model proposed by Ma et al. (2016), an application of the Contact and Channel Model discussed by Albers et al. (2011), and the parameter-based approach by Rouibah and Caskey (2003).

\subsection{Single vs. multiple domains}

Many change propagation prediction models focus on the structure of a design and do not explicitly take its behaviour into account. While this allows for a concise representation, it is also limited because repercussions of design change do not only affect the physical aspects of a design, such as dimensions, but also impact other aspects such as functionality and requirements. Taking this into consideration some authors have proposed multi-domain models that help trace design change to requirements and functions. Because they include requirements and functions explicitly, these models may help ensure product compliance and conformance following a change.

Most relevant to this paper, Ahmad et al. (2013) developed a framework called the ISF which uses a multi-domain approach to model the product design. Ahmad et al. (2013) argue that a detailed assessment of how a change will propagate requires information about why the design is the way it is. The ISF accordingly uses four domains (layers) to model a design, namely Requirements, Functions, Components, and Detail Design Process, to model the information structures relating to a design and its creation. Each layer is connected to each other so that change propagation can be traced between them. The ISF was shown to be useful in experiments by Ahmad et al. (2013) and opens many areas for further work. It provides a foundation for the research described in this paper.

\section{The design process and change management}

The way a change propagates through an artefact arguably is influenced by how it was designed in the first place. The proposed model aims to help investigate and assess change propagation by capturing and retracing the design process. This section discusses key features of the design process with a view to identifying what needs to be captured in such a model.

\subsection{Iterations in design}

The design process has been described by many authors. Some, like French (1985), describe design as a stage-based process. Authors such as Hubka and Eder (1988), complement this perspective with more detailed theories related to the constitution of mechanical systems and their operations. One common feature of all models is to emphasise the iterative nature of design. Iterations in design can have different characteristics according the situation (Wynn and Eckert, 2017). Of particular interest in this paper, designers often need to work under incomplete information, making estimates of unknown parameters which can then be used to make progress. If assumptions later prove inappropriate, some work may need to be redone. The gap between the estimated parameter and parameter embodied in the design is decreased by repeated iterations, moving towards an optimised solution (Browning, 2001). 


\subsection{Multiple solutions are possible}

Another aspect of the (re)design process is the possibility of multiple solution concepts, more than one of which may be made to work. Decision making between alternatives considers various constraints such as cost, manufacturing capabilities, space considerations, binding codes and standards, legislations etc. Analysis techniques such as the Pugh controlled convergence method and morphological analysis stipulate systematic generation and evaluation of concepts considering multiple possible solutions. In some cases, decisions are informed by such methods while in others, decisions are based on engineering experience and judgement.

\subsection{Design decisions and design history}

Design decisions form a valuable part of the overall design knowledge base. It is our view that knowing the rationale behind the design and the design process history may assist the accurate assessment of change propagation. For example, a change may be initiated to mitigate a defect, the cause of which may be traced back to a decision taken during the original design process. Therefore, when a change occurs, the designers revisit the decisions made during the original design process. This may be represented and possibly supported through the capture of design rationale and design history. Design rationale refers to the reasoning behind decisions made in the design process. Design history, on the other hand, refers to the process followed when the design was created, for example, the sequence of operations, iterations, etc.

In this paper we argue that a representation of design rationale and design history of the original design process can help to understand the redesign process, and information regarding the design alternatives considered and design decisions made during the original design process could provide redesign guidance by allowing a designer to reselect among those alternatives. Moreover, original design rationale and decisions also should be considered during the redesign process, especially when multiple designers contribute and may benefit from explicit representation of the reasoning behind each other's decisions.

Design rationale representation approaches have been developed since the 1970s. Models of design rationale capture have often been classified as approach-oriented and feature-oriented (Regli et al., 2000), argumentative-based and intent-driven (Liu and $\mathrm{Hu}, 2013$ ). Methods such as the Issue-Based Information System (IBIS) (Kunz and Rittel, 1970) are also relatively well known. DRED (Bracewell et al., 2009; Aurisicchio et al., 2012, 2013) is another platform where design rationale capture has been implemented. Similarly, authors have proposed approaches which capture the history of a design process. Approaches such as DRL (Lee and Lai, 1991) and PHI (McCall, 1991) are well known. Although design rationale and design history capture have been under research since 1970s, these concepts have not explicitly been integrated into recent work to support change propagation assessment and redesign.

\subsection{Summary: Need for a structured framework to capture relevant information}

Overall, the information used and created during a design process is of varied nature. For example, it may concern requirements, the physical and functional attributes of the designed artefact, the design history and rationale, CAD data, and analysis data. The links between such information are important in determining how design change propagates. A framework to assist in capturing and structuring the information network would be helpful to support propagation assessment and redesign, and would assist fundamental research into what causes change propagation. In this paper a new multidomain model is proposed towards addressing this gap.

\section{The proposed model}

Study of the literature and concepts discussed above show that there is a need for a new model which should have the following features:

1. It should incorporate relationships between design decisions impacting different components.

2. It should be able to capture the design history and rationale.

3. It should be able to record alternatives evaluated during the design process. 
4. It should provide for the iterative nature of design.

5. The constraints considered during design should be incorporated.

We set out to develop a model that addresses these points, and to assess whether this micro-level model can support change impact assessment and the redesign process more effectively than existing approaches. The following subsections lay down a theoretical basis for the proposed model, defining the terms used.

The model is based on the concept that a design problem can be decomposed into a set of interconnected subproblems. The designer iteratively addresses these subproblems. Each time a sub-problem is taken up or revisited, we propose the designer's activity can be captured by filling in a set of fields representing a generic micro-level design step. During the design process, as each sub-problem iteration is completed, the fields of a generic template are populated with information to capture what happened. This can later be used to assess the impact of a redesign, specifically how, and why, changes propagate.

The template is shown in Figure 1 and the fields are defined in Table 1. The next section explains and illustrates the knowledge capture approach with an illustrative example.

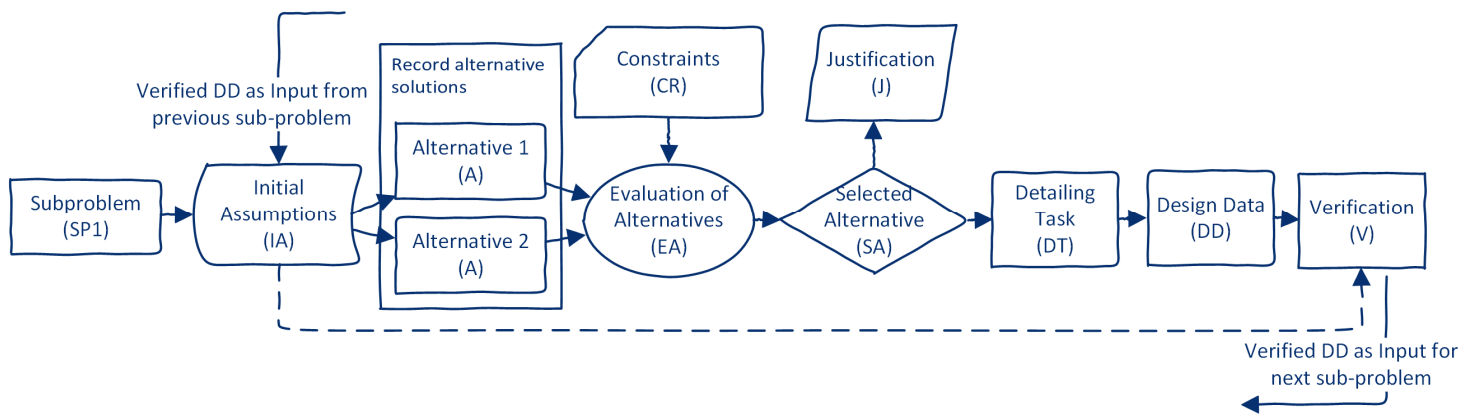

Figure 1. Overview of the template for capturing a design step to aid change propagation analysis and redesign

\subsection{Illustrative example}

A simplified desk-based case study was undertaken by the first author to illustrate and assess the model. The case study is based on a project done by Part II undergraduate students during a mechanical engineering design course at the University of Auckland. The case involves a mixture of machine element selection and shaft calculations, in order to design a winch system under load and speed requirements while being constrained by space. Although allowing for different solutions, the problem is essentially routine.

In this case, the first author developed a design solution consisting of two co-axial shafts joined together using a flexible coupling (Figure 2). In the design, one winch drum was mounted on each shaft. A 3phase motor with speed reduction was selected to run the shafts at the required speed. A chain drive system was used to transfer power from the motor to the shaft. Other auxiliary components such as bearings, taperlock bushes, keyways, lubrication and support were also included in the design.

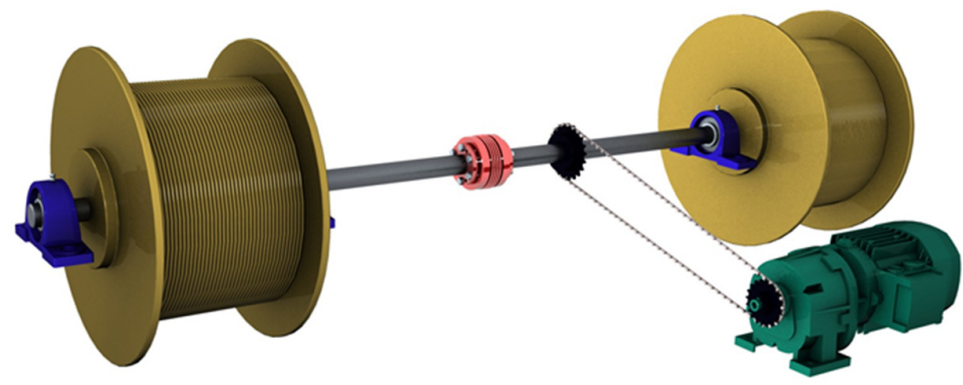

Figure 2. Components of the mechanical system considered for the case study 
The propagation model was constructed in a series of layers, interconnected by coded hyperlinks (see Table 1 for key). Requirements were extracted from the design brief and used to construct the Requirements Layer as stipulated by Ahmad et al. (2013) (Figure 3). The Function Layer (Figure 4) and Component Layer (Figure 5) were similarly developed as stipulated by Ahmad et al. (2013). Layers in the model are connected using hyperlinks, with coding scheme explained in Table 1. For example, in Figure 4, the function "F2: Distribute electricity" has 2 hyperlink connections. The hyperlink labelled "R5" indicates an incoming connection from object R5 on the requirements layer (Figure 3) and similarly the hyperlink "C2" indicates a connection to an object on the components layer (Figure 5).

Table 1. Code scheme and field descriptions used in the framework

\begin{tabular}{|c|c|c|}
\hline Code & Entity & Description \\
\hline N/A & Design step & A single step in the design process, as depicted in Figure 1 \\
\hline R1, R2, etc. & Requirement & $\begin{array}{l}\text { Criteria a product should fulfil, in other words, the basic } \\
\text { need for the product to exist (Ahmad et al., 2013). }\end{array}$ \\
\hline $\mathrm{F} 1, \mathrm{~F} 2$, etc. & Function & $\begin{array}{l}\text { Intermediaries between requirements and embodiment of a } \\
\text { design (Ahmad et al., 2013). }\end{array}$ \\
\hline $\mathrm{C} 1, \mathrm{C} 2$, etc & Component & $\begin{array}{l}\text { The entities necessary to realise the functions in the } \\
\text { physical domains, or "function carriers" (Hubka and Eder, } \\
\text { 1988). }\end{array}$ \\
\hline $\mathrm{SP} 1, \mathrm{SP} 2$, etc & Sub-problem & $\begin{array}{l}\text { Subdivisions of the design problem which are each } \\
\text { resolved by one design step. }\end{array}$ \\
\hline DTO1, DTO2, etc. & Design Task Objective & The goal to be achieved in terms of parameters of a design. \\
\hline CR1, CR2, etc & Constraints & $\begin{array}{l}\text { The quantitative and qualitative factors which put limiting } \\
\text { conditions on the design decisions. }\end{array}$ \\
\hline IA 1 , IA2, etc & Initial Assumptions & $\begin{array}{l}\text { Assumptions made at the beginning of a design step, due } \\
\text { to lack of necessary information required to proceed. }\end{array}$ \\
\hline $\mathrm{A} 1, \mathrm{~A} 2$, etc & Alternatives & $\begin{array}{l}\text { All alternative design solutions considered in the design } \\
\text { step. }\end{array}$ \\
\hline EA1, EA2, etc & $\begin{array}{l}\text { Evaluation of } \\
\text { Alternatives }\end{array}$ & $\begin{array}{l}\text { How the alternatives } \mathrm{A} 1, \mathrm{~A} 2 \text {, etc. are evaluated among } \\
\text { each other considering applicable constraints. }\end{array}$ \\
\hline $\mathrm{SA} 1, \mathrm{SA} 2$, etc & Selected Alternative & The single chosen alternative. \\
\hline $\mathrm{J} 1, \mathrm{~J} 2$, etc & Justification & $\begin{array}{l}\text { The reasons underlying the chosen selection from } \\
\text { alternatives. }\end{array}$ \\
\hline DT1, DT2, etc & Detailing Task & $\begin{array}{l}\text { The tasks undertaken (such as design calculations, FEA } \\
\text { etc) to detail the attributes of the selected alternative. }\end{array}$ \\
\hline DD1, DD2, etc & Design Data & The design information created during the design step. \\
\hline $\mathrm{V} 1, \mathrm{~V} 2$, etc & Verification & $\begin{array}{l}\text { The initial assumptions made are verified against Design } \\
\text { Data. Provides input data to next sub-problem. }\end{array}$ \\
\hline
\end{tabular}



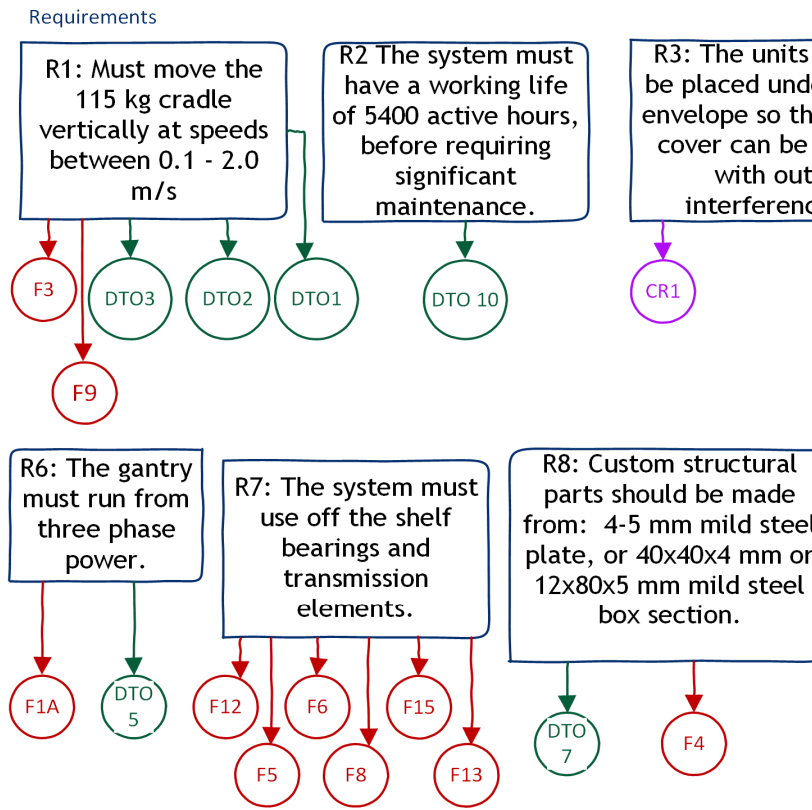

Figure 3. Requirements layer for the case study; Circles show hyperlinks (as per codes in Table 1) to objects on other layers

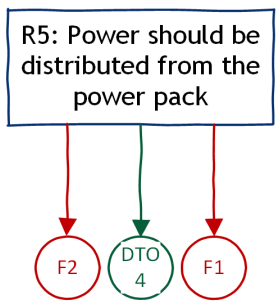

R10: Two cylindrical winch drums must be placed to raise and lower the cradle cables

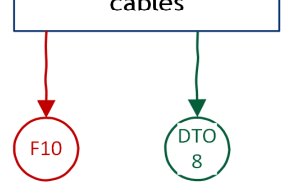

\subsubsection{Modified detail design layer}

Subsequently, the detail design process layer was populated using the expanded template of Figure 1. This was done concurrently while completing the design process. A list of design task objectives was developed by elaborating each requirement. For example, the requirement "R1: Must move the $115 \mathrm{~kg}$ cradle vertically at speeds between $0.1-2 \mathrm{~m} / \mathrm{s}$ " (appearing on Figure 3 ) led to the design task objectives "DTO1: Maximum lift weight:115kg.", "DTO2: Maximum Lift Speed:2m/s" and "DTO3: Minimum Lift Speed: $0.1 \mathrm{~m} / \mathrm{s} "$ (appearing on Figure 6).

For the case study design process, eleven sub-problems were addressed in total. Examples discussed here are "SP1: Select motor from catalogue" and "SP2: Design and select speed reduction mechanism" (at the top of Figure 6). The sub-problems were each linked to the component(s) involved. For example, as shown in Figure 6, SP1 was connected to "C2: Motor" and "C4: Gearbox". Having identified the sub-problems, the detail design of the system was completed to address these subproblems, and simultaneously, the detail design layer was constructed (first 2 sub-problems shown in Figure 6). The sub-problems were each addressed in sequence. In design, different sequences are typically possible. The model stipulates recording of the sequence chosen by the designer, including iterations.

To start on a sub-problem, the "known inputs" and "unknown inputs" were first identified. Assumptions for the latter were made in order to proceed. For example, in order to proceed with the sub-problem "SP1: select motor from catalogue", a specific design speed was needed, whereas only a range was available (as shown by the links from DTO2 and DTO3 to SP1 in Figure 6). Therefore, design proceeded with the assumption that designing for the highest speed would cover the range as specified. This is captured as Initial Assumptions (IA1) in Figure 6. The Design Task Objectives (DTO1 to DTO5, DTO8 and DTO9) and Initial Assumptions (IA1) were then used as input to identify potential alternative solutions. In this case, a motor could be a geared unit or a motor with a separate gearbox. The considered alternatives were recorded (A1, A2 and A3 in Figure 6). 


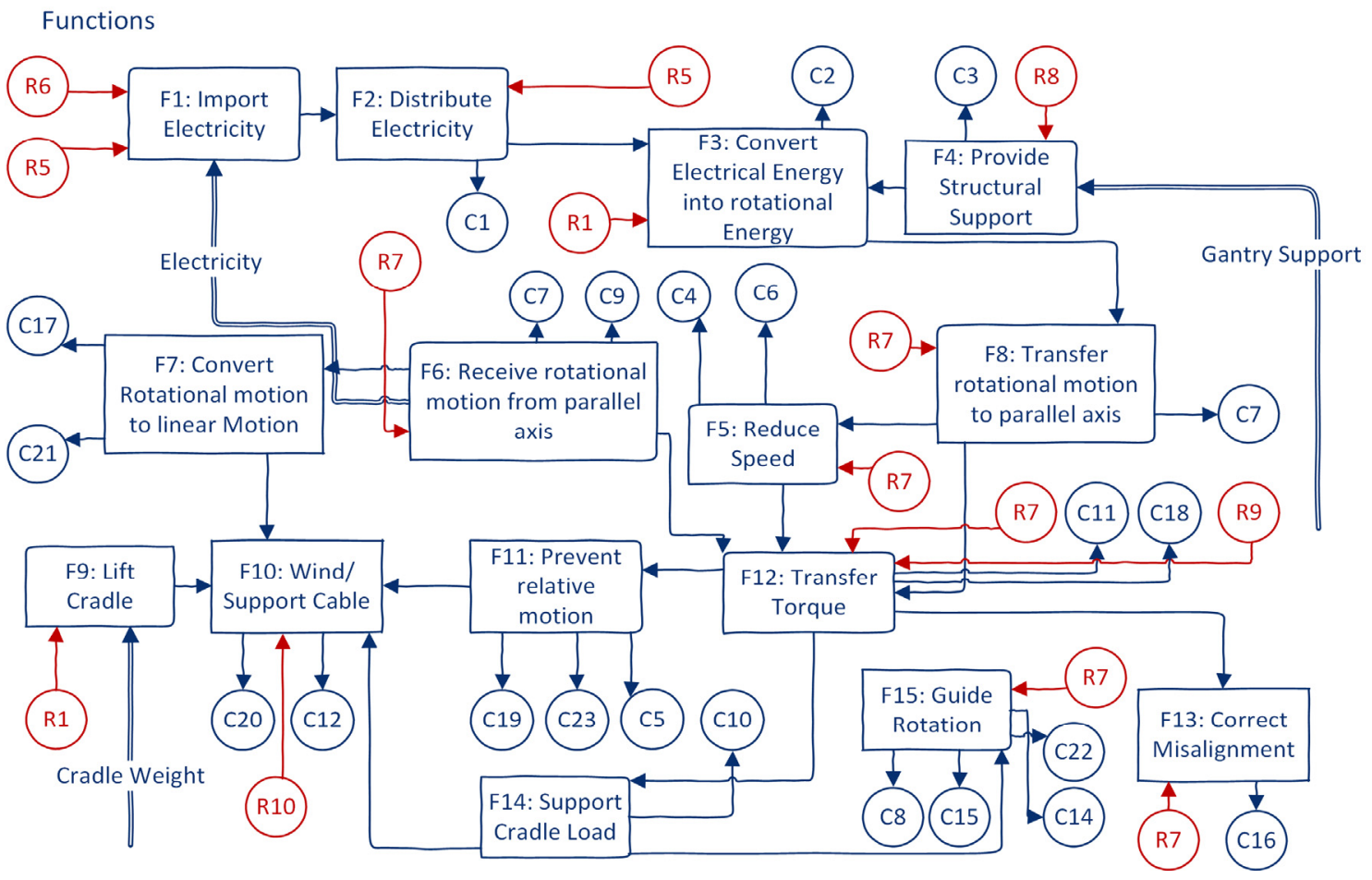

Figure 4. Functions layer for the case study;

Coded hyperlinks to Requirements layer (Figure 3) and Components layer (Figure 5)

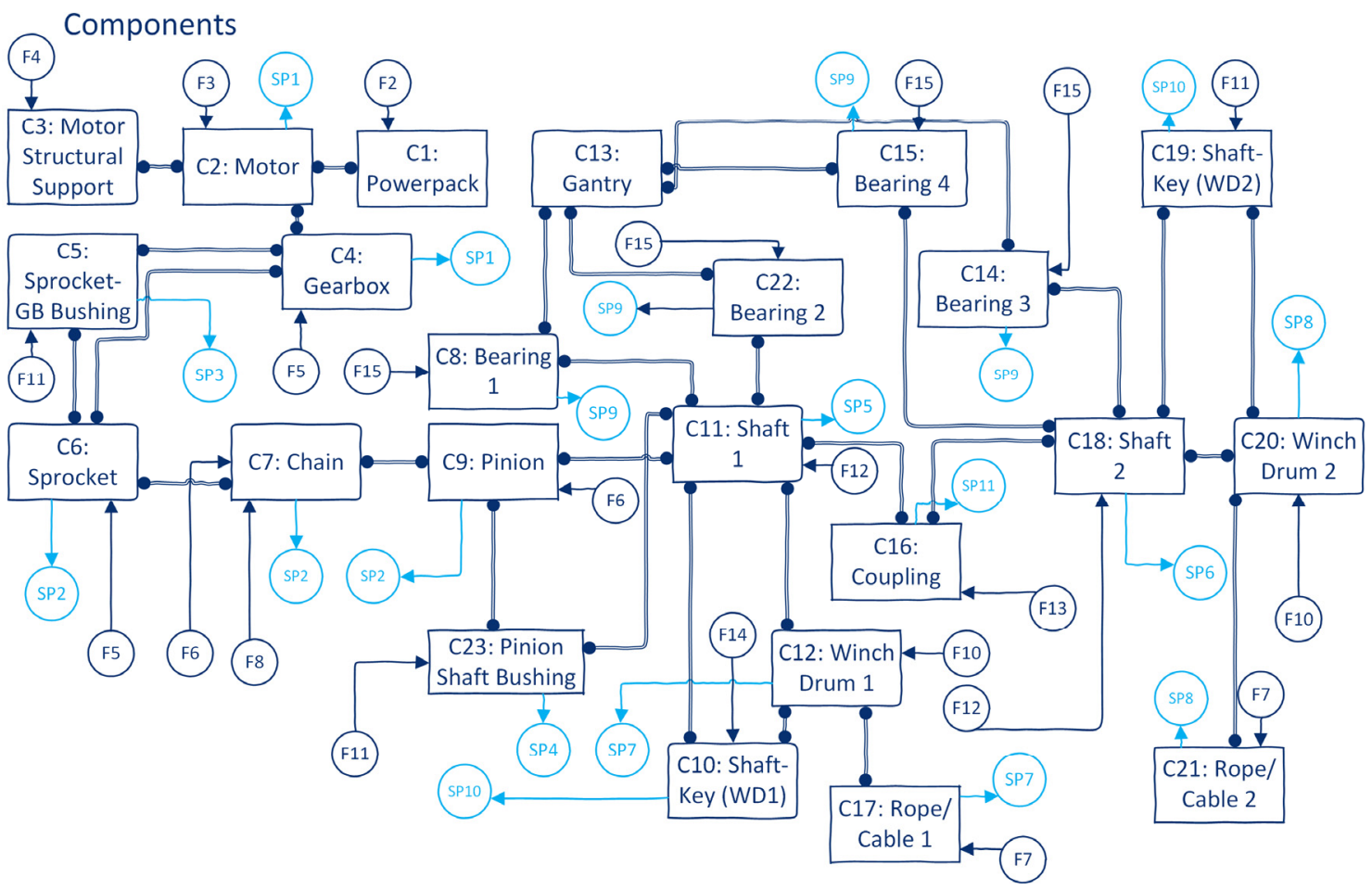

Figure 5. Component layer for the case study;

Coded hyperlinks indicate connections from Function layer (Figure 4) and to Detail Design Process layer (Figure 6) 


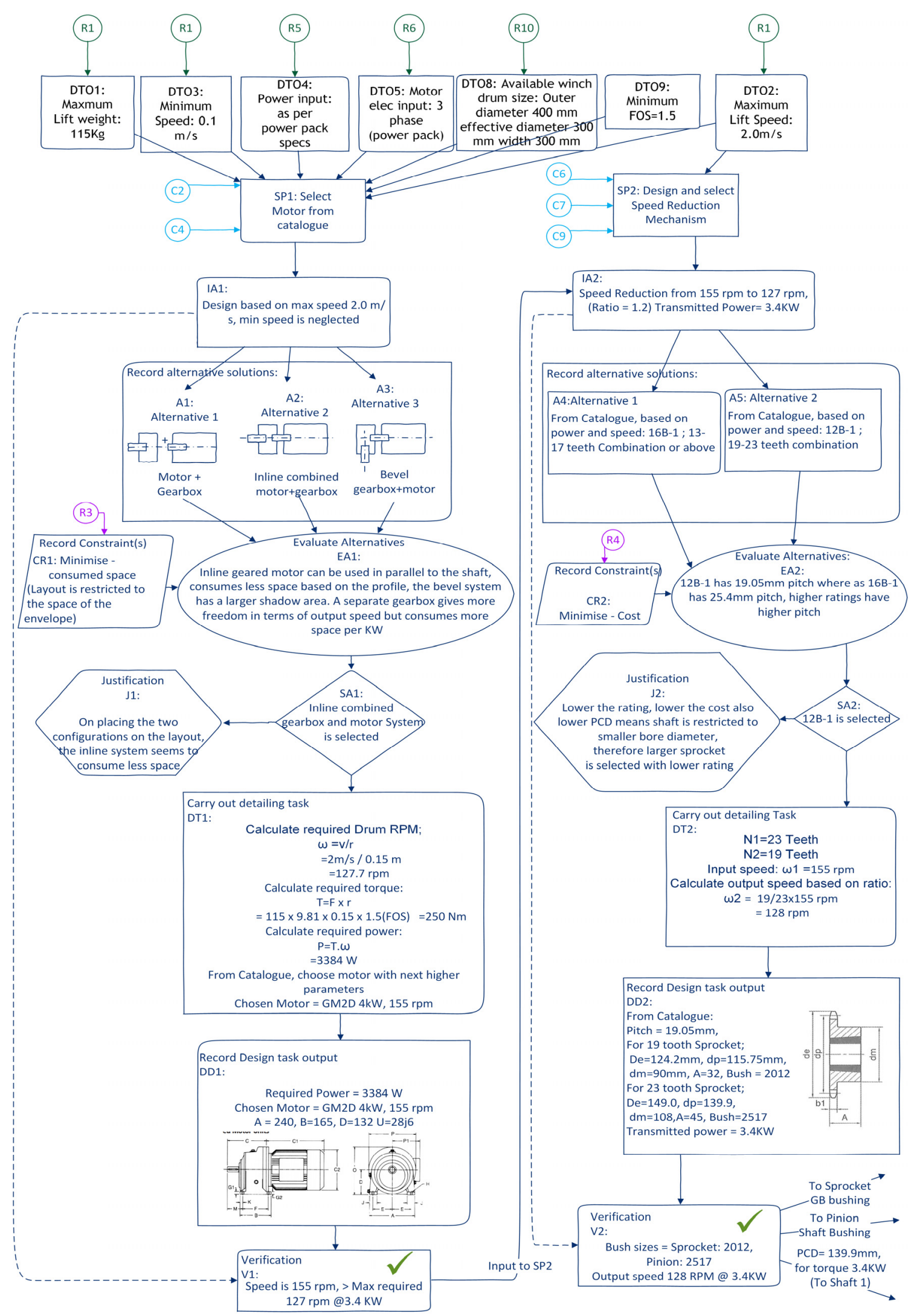

Figure 6. Detail of the first two (of eleven) sub-problems of the case study 
Then, a decision was needed to select a single option to take forward. Constraints (i.e. criteria to be considered in the decision) were identified and recorded. In this case, a key constraint in motor selection was "CR1: minimise the space required". The alternatives were evaluated, and the comparison was recorded as Evaluation of Alternatives (EA1). After making a decision, the outcome was recorded (SA1) along with a brief textual description of the decision rationale (J1). Finally, detailing tasks were carried out as necessary (in this case, selection of a specific motor, captured in DT1) and the outputs captured (field DD1, here: catalogue code of the motor, mounting details, weight, output speed, torque etc.). These outputs were compared to the initial assumption IA1 in Verification V1 using a feedforward, found to be acceptable, and became available as known inputs for subsequent subproblems. As an illustration, the first two sub-problems recorded in the detail design layer are presented in Figure 6.

One of the features of the model is to store historical design data and rationale to support post-change redesign. In the illustrative example, during the original design process of sub-problem 1, the motor was selected based on certain design decisions in addition to the calculations made, in this case the availability of space constraint. During redesign, the alternatives evaluated are explicitly captured and hence can be revisited. Two distinct cases were found to be possible:

1. An alternative earlier evaluated but discarded proves to be a viable alternative candidate.

2. The sub-problem solution must be redesigned completely.

Both these cases are discussed below.

Once the redesign process is initiated, the detail design sequence is reactivated starting with the changed information. The designer retraces the same steps captured in the model, although the information might be different. As they proceed, new information is generated, and new instances of the design sequence are added as another layer on top of the old layer as shown in Figure 7 (left). Each new instance of an object replaces the old one, though the old one is retained as part of the design history and not discarded. It is anticipated that this history may be relevant for analysing future changes, although the means by which the information might be searched and used, and its consistency managed, has not yet been researched in depth (this is one of our main areas for future research). The redesign propagation process continues (and layers of instances are added to the model), until a step is reached where further propagation does not occur, or a design step is reached where no solution is found amongst the existing alternatives. In that case, the situation moves towards case 2 , where a complete redesign is undertaken.

In case of a complete redesign of the sub-problem solution is needed, the input data, assumptions and constraints are readily available in the model, which should help to inform and direct the redesign process. An example of this can be found in Figure 7 (right), representing a case where the shaft had to be completely redesigned and needed fresh calculations.

\subsection{Change propagation in the model}

The detailed information provided by the approach lays a foundation for in-depth analysis of the causes of change propagation for a modelled design. In particular the model makes explicit the constraints on each sub-problem at the time a solution was found. This clarifies the window in which the redesign task can be undertaken without causing propagation.

For example, in the case study, if the shafts need to be redesigned it can be seen what other subproblems constrain it and are dependent on it. In the case illustrated (Figure 7 right), the shaft design is dictated by the drum size on one end (because the drum's size is the basis for the drum's weight and required torque, which in turn determines the minimum shaft diameter to prevent failure, $11 \mathrm{~mm}$ in this case). On the other hand, the shaft's maximum diameter is determined by the other components dependent on it such as the sprocket's bore ( $48 \mathrm{~mm}$ in this case), which is to be mounted on the shaft. All these relationships are explicit in the model. This could potentially assist the redesign process by guiding the design engineer to stay within the window of the original design if possible, thereby not disturbing the other dependent components. When the constraints causing propagation are made clear this may also prompt consideration of new design options to avoid knock-on effects, in this case perhaps by creating ribs on the shaft to give it additional strength without changing the nominal diameter. 
In many cases a buffer zone (threshold) exists, up to which the change would not cause any knock-on effects. This happens due to the design's ability to absorb changes, which could be because of reasons such as a factor of safety, selection of a component of nearest higher rating, etc. An example of such an occurrence can be seen in Figure 6, sub-problem 1. For the motor, the manufacturer offers a range of discrete options, so in DT1, the motor selected was the lowest motor power that exceeded the power requirement that was derived from various Design Task Objectives (DTOs) and Requirements (Rs). In this specific case there is a buffer of $616 \mathrm{~W}$ (selected motor being $4 \mathrm{~kW}$ and required power being $3384 \mathrm{~kW}$ ) built into the design. This creates a buffer zone within which the design can be varied without propagating further. The model explicitly captures how any change to the load requirement will not propagate to cause change to the motor unless it exceeds the rating (although other components might need to be changed).

\subsection{Summary of example}

The example shows how the framework offers additional insight to existing approaches, such as those summarised in Section 2. In particular the framework offers potential for detail guidance for redesign. By capturing redesign processes using this model, we intend to investigate the causes of change propagation in depth to gain better theoretical understanding, which might ultimately lead to better pragmatic support tools.

The developed approach has limitations, most obviously the problems associated with handling such large amounts of data. The illustrative example was limited in scale and the amount of data would increase substantially with the scope and complexity of the design. It can also be anticipated that elicitation and visualisation of data in the model will be difficult as more complex applications are trialled. Finally, the design example presented in this paper represents a routine design situation, which has been the focus of the research thus far. We plan to investigate applicability of the framework to other kinds of design involving more novelty, and involving architectural decisions that would involve change to the Component and/or Function layers of the model.

\section{Discussion and conclusion}

The proposed model offers a micro-level process-oriented perspective on design change propagation. It may help to understand and research the causes of change propagation, guide the redesign process, and analyse in greater detail the mechanisms through which changes propagate or are absorbed. The research is currently in relatively early stages (the first year of a $\mathrm{PhD}$ project) but we believe the approach offers a new perspective on change propagation and highlights opportunities for further work towards understanding the change propagation process and, possibly, towards a pragmatic support tool.

One key issue for future work is to investigate how much detail should be captured and what happens to the model behaviour when the abstraction level is increased. For some applications, it might be appropriate to focus on how the change propagates on the sub-systems level rather than on the parametric level. Another question is the behaviour of the model when the effects of a change fall in the buffer zone of the design as discussed in Section 4.2. We aim to investigate how buffers can be incorporated into the model and therefore, investigate the circumstances under which a change will propagate or be absorbed-as well as the best placement of margin during a design process to make a design less susceptible to future change propagation. It is planned that further case studies involving different design scenarios of higher complexities will be undertaken to investigate these issues arising from the preliminary case study. If successful, the proposed approach's possible integration with $\mathrm{CAD} / \mathrm{PDM} / \mathrm{PLM}$ and other knowledge management software may be investigated.

To summarize, a new model for capturing design information with an aim to aid change propagation assessment and subsequent redesign has been proposed. The preliminary case study shows promising results towards investigating change propagation using systematic capture of design history and design rationale. A preliminary case study gave rise to many questions, which are planned to be investigated via further case studies. Ultimately it is hoped to use these insights to develop a pragmatic support tool. 

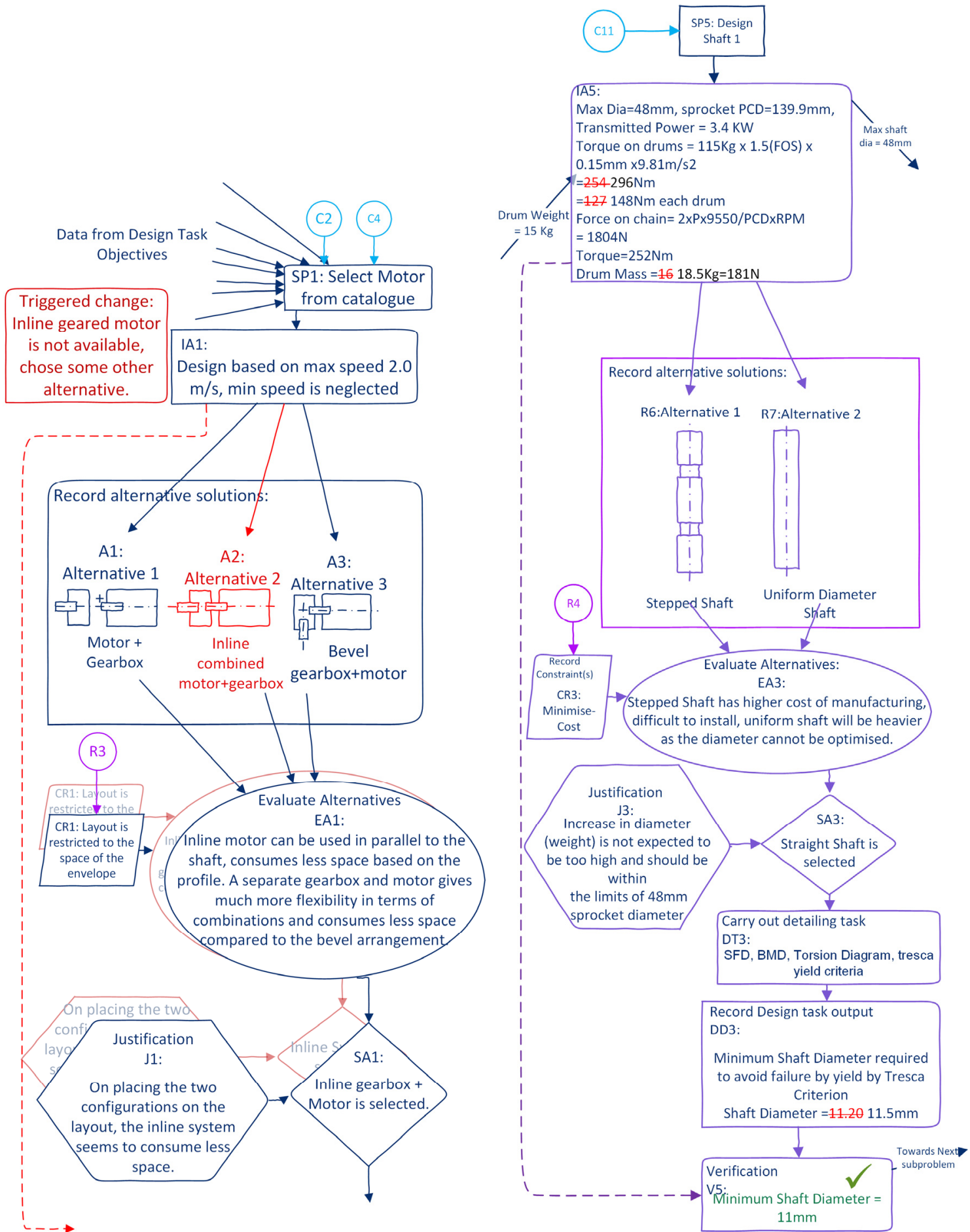

Figure 7. Alternative as a redesign choice (left). Complete redesign as a solution to a sub-problem (right)

\section{References}

Ahmad, N., Wynn, D.C. and Clarkson, P.J. (2013), "Change impact on a product and its redesign process: a tool for knowledge capture and reuse", Research in Engineering Design, Vol. 24 No. 3, pp. 219-244. https://doi.org/10.1007/s00163-012-0139-8 
Albers, A., Braun, A., Sadowski, E., Wynn, D.C., Wyatt, D.F. and Clarkson, P.J. (2011), "System Architecture Modeling in a Software Tool Based on the Contact and Channel Approach (C\&C-A)", Journal of Mechanical Design, Vol. 133 No. 10, p. 101006. https://doi.org/10.1115/1.4004971

Aurisicchio, M., Bracewell, R. and Armstrong, G. (2012), "The Function Analysis Diagram", ASME 2012 International Design Engineering Technical Conferences and Computers and Information in Engineering Conference, Volume 7: 9th International Conference on Design Education; 24th International Conference on Design Theory and Methodology, August 12-15, 2012, pp. 849. https://doi.org/10.1115/DETC2012-70944

Aurisicchio, M., Bracewell, R. and Armstrong, G. (2013), "The function analysis diagram: Intended benefits and coexistence with other functional models", Artificial Intelligence for Engineering Design, Analysis and Manufacturing, Vol. 27 No. 3, pp. 249-257. https://doi.org/10.1017/S0890060413000255

Bracewell, R., Gourtovaia, M., Moss, M., Knott, D., Wallace, K. and Clarkson, P.J. (2009), "DRed 2.0: a method and tool for capture and communication of design knowledge deliberated in the creation of technical products", DS 58-6: Proceedings of ICED 09, the 17th International Conference on Engineering Design, Vol. 6, Design Methods and Tools (pt. 2), Palo Alto, CA, USA, August 24 - 27, 2009.

Browning, T.R. (2001), "Applying the design structure matrix to system decomposition and integration problems: a review and new directions", IEEE Transactions on Engineering Management, Vol. 48 No. 3, pp. 292-306. https://doi.org/10.1109/17.946528

Clarkson, P.J., Simons, C. and Eckert, C. (2004), "Predicting Change Propagation in Complex Design”, Journal of Mechanical Design, Vol. 126 No. 5, pp. 788-797. https://doi.org/10.1115/1.1765117

French, M.J. (1985), “Introduction”, In: French, M.J. (Ed.), Conceptual Design for Engineers, Springer, Berlin, Heidelberg, pp. 1-12. https://doi.org/10.1007/978-3-662-11364-6

Fricke, E., Gebhard, B., Negele, H. and Igenbergs, E. (2000), "Coping with changes: Causes, findings, and strategies”, Systems Engineering, Vol. 3 No. 4, pp. 169-179. https://doi.org/10.1002/15206858(2000)3:4<169::AID-SYS1>3.0.CO;2-W

Hubka, V. and Eder, W.E. (1988), Theory of Technical Systems, Springer Nature. https://doi.org/10.1007/978-3642-52121-8

Kunz, W. and Rittel, H.W. (1970), Issues as elements of information systems, Institute of Urban and Regional Development, University of California Berkeley, California.

Lee, J. and Lai, K. (1991), “What's in Design Rationale?”, Human-Computer Interaction, Vol. 6 No. 3-4, pp. 251280. https://doi.org/10.1080/07370024.1991.9667169

Liu, J. and Hu, X. (2013), "A reuse oriented representation model for capturing and formalizing the evolving design rationale", Artificial Intelligence for Engineering Design, Analysis and Manufacturing, Vol. 27 No. 4 , pp. 401-413. https://doi.org/10.1017/S0890060413000395

Ma, S., Jiang, Z. and Liu, W. (2016), "A design change analysis model as a change impact analysis basis for semantic design change management", Proceedings of the Institution of Mechanical Engineers, Part C: Journal of Mechanical Engineering Science, Vol. 231 No. 13, pp. 2384-2397. https://doi.org/10.1177/0954406216633035

McCall, R.J. (1991), "PHI: a conceptual foundation for design hypermedia”, Design Studies, Vol. 12 No. 1, pp. 30-41. https://doi.org/10.1016/0142-694X(91)90006-I

Regli, W.C., Hu, X., Atwood, M. and Sun, W. (2000), “A Survey of Design Rationale Systems: Approaches, Representation, Capture and Retrieval", Engineering with Computers, Vol. 16 No. 3, pp. 209-235. https://doi.org/10.1007/PL00013715

Rouibah, K. and Caskey, K.R. (2003), "Change management in concurrent engineering from a parameter perspective", Computers in Industry, Vol. 50 No. 1, pp. 15. https://doi.org/10.1016/S0166-3615(02)00138-0

Terwiesch, C. and Loch, C.H. (1999), "Managing the Process of Engineering Change Orders: The Case of the Climate Control System in Automobile Development", Journal of Product Innovation Management, Vol. 16 No. 2, pp. 160-172. https://doi.org/10.1111/1540-5885.1620160

Wynn, D.C. and Eckert, C.M. (2017), "Perspectives on iteration in design and development", Research in Engineering Design, Vol. 28 No. 2, pp. 153-184. https://doi.org/10.1007/s00163-016-0226-3

Arindam Brahma, Doctoral Candidate

The University of Auckland, Mechanical Engineering

Level-9, Tower Block, 1010 Auckland, New Zealand

Email: abra248@aucklanduni.ac.nz 